\title{
High-frequency dynamics of wave localization
}

\author{
C W J Beenakker and $\mathrm{K} \mathrm{J} \mathrm{H}$ van Bemmel \\ Instituut-Lorentz, Universiteit Leiden, PO Box 9506, 2300 RA Leiden The Netherlands \\ P W Brouwer \\ Laboratory of Atomic and Soldd State Phystcs Cornell Unversity, Ithaca, New York 14853 \\ (Recerved 3 September 1999)
}

\begin{abstract}
We study the effect of localization on the propagation of a pulse through a multımode disordered waveguide The correlator $\left\langle u\left(\omega_{1}\right) u^{*}\left(\omega_{2}\right)\right\rangle$ of the transmitted wave amplitude $u$ at two frequencies differing by $\delta \omega$ has for large $\delta \omega$ the stretched exponentral tal $\propto \exp \left(-\sqrt{\tau_{D} \delta \omega / 2}\right)$ The time constant $\tau_{D}=L^{2} / D$ is given by the diffusion coefficient $D$, even if the length $L$ of the waveguide is much greater than the localization length $\xi$ Localization has the effect of multiplying the correlator by a frequency-independent factor $\exp (-L / 2 \xi)$, which disappears upon breakıng tıme-reversal symmetry [S1063-651X(99)50412-1]

PACS number(s) $4225 \mathrm{Dd}, 4225 \mathrm{Bs}, 7215 \mathrm{Rn}, 9130$-f
\end{abstract}

The frequency spectrum of waves propagating through a random medium contains dynamical information of interest in optics [1], acoustics [2], and seismology [3] A fundamental issue is how the phenomenon of wave localization [4] affects the dynamics The basic quantity is the correlation of the wave amplitude at two frequencies differing by $\delta \omega \mathrm{A}$ recent microwave experiment by Genack et al [5] measured this correlation for a pulse transmitted through a waveguide with randomly positioned scatterers The waves were not localızed in that experiment, because the length $L$ of the waveguide was less than the localization length $\xi$, so the correlator could be computed from the perturbation theory for diffusive dynamics [6] The characteristic time scale in that regime is the time $\tau_{D}=L^{2} / D$ it takes to diffuse (with diffusion coefficient $D$ ) from one end of the waveguide to the other According to diffusion theory, for large $\delta \omega$ the cor1elatol decays $\propto \exp \left(-\sqrt{\tau_{D} \delta \omega / 2}\right)$ with time constant $\tau_{D}$

What happens to the high-frequency decay of the corielator if the waveguide becomes longer than the localization length? That is the question addiessed in this Rapid Communication Our prediction is that, although the conelator is suppressed by a factor $\exp (-L / 2 \xi)$, the time scale for the decay remains the diffusion time $\tau_{D}$, even if diffusion is only possible on length scales $\ll L$ The exponentral suppression factor disappears if time-reversal symmetry is broken (by some magneto-optical effect) Our analytical results are based on the formal equivalence between a frequency shift and an imaginary absorption rate, and are supported by a numerical solution of the wave equation

We consider the propagation of a pulse through a disordered waveguide of length $L$ In the frequency domain the transmission coefficient $t_{n m}(\omega)$ gives the ratio of the transmitted amplitude in mode $n$ to the incident amplitude in mode $m$ (The modes are normalized to cany the same flux ) We seek the correlator $C(\delta \omega)=\left\langle t_{n m}(\omega+\delta \omega) t_{n m}^{\psi}(\omega)\right\rangle$ (The brackets \langle\rangle denote an average over the disorder ) We assume that the (positive) frequency inciement $\delta \omega$ is sufficiently small compared to $\omega$ that the mean fiee path $l$ and the number of modes $N$ in the wavegurde do not vary appreciably, and may be evaluated at the mean frequency $\omega[7] \mathrm{We}$ also assume that $l \gg c / \omega$ (with $c$ the wave velocity) The localization length is then given by [8] $\xi=(\beta N+2-\beta) l$, with $\beta=1$ (2) in the presence (absence) of time-reversal symmetry For $N \gg 1$ the localization length is much greater than the mean fiee path, so that the motion on length scales below $\xi$ is diffusive (with diffusion coefficient $D$ )

Our appioach is to map the dynamic problem without absorption onto a static problem with absorption [9] The mapping is based on the analyticity of the transmission amplitude $t_{n m}(\omega+t y)$, at complex frequency $\omega+\imath y$ with $y$ $>0$, and on the symmetry relation $t_{n m}(\omega+\imath y)=t_{n m}^{*}(-\omega$ $+t y)$ The product of tiansmission amplitudes $t_{n m}(\omega$ $+z) t_{n m}(-\omega+z)$ is therefore an analytic function of $z$ in the upper half of the complex plane If we take $z$ eal, equal to $\frac{1}{2} \delta \omega$, we obtain the product of transmission amplitudes $t_{n m}\left(\omega+\frac{1}{2} \delta \omega\right) t_{n m}^{*}\left(\omega-\frac{1}{2} \delta \omega\right)$ considered above [the difference with $t_{n m}(\omega+\delta \omega) t_{n m}^{*}(\omega)$ being statistically irrelevant for $\delta \omega \ll \omega]$ If we take $z$ imaginary, equal to $t / 2 \tau_{a}$, we obtain the transmission probability $T=\left|t_{n m}\left(\omega+\imath / 2 \tau_{a}\right)\right|^{2}$ at frequency $\omega$ and absorption tate $1 / \tau_{a}$ We conclude that the conelator $C$ can be obtained from the ensemble average of $T$ by analytic continuation to imaginary absorption rate,

$$
C(\delta \omega)=\langle T\rangle \text { for } 1 / \tau_{a} \rightarrow-l \delta \omega
$$

Two remaiks on this mapping (1) The effect of absorption (with tate $\left.1 / \tau^{*}\right)$ on $C(\delta \omega)$ can be included by the substitution $1 / \tau_{a} \rightarrow-\imath \delta \omega+1 / \tau^{*}$ This is of importance for compaison with experiments, but here we will for simplicity ignoie this effect (il) Higher moments of the product $\mathcal{C}$ $=t_{n m}\left(\omega+\frac{1}{2} \delta \omega\right) t_{n m}^{*}\left(\omega-\frac{1}{2} \delta \omega\right)$ are related to higher moments of $T$ by $\left\langle\mathcal{C}^{p}\right\rangle=\left\langle T^{p}\right\rangle$ for $1 / \tau_{a} \rightarrow-{ }_{l} \delta \omega$ This is not sufficient to deter mine the entire probability disti ibution $P(\mathcal{C})$, because moments of the form $\left\langle\mathcal{C}^{p} \mathcal{C}^{* q}\right\rangle$ cannot be obtained by analytic continuation [10]

To check the validity of this approach and to demonstiate how effective it is we consider briefly the case $N=1$ A disordered single-mode waveguide is equivalent to a geometry of parallel layers with random variations in composition and thickness Such a randomly stratıfied medium is studied 
in seismology as a model for the subsurface of the Earth [3] The correlator of the reflection amplitudes $K(\delta \omega)$ $=\left\langle r(\omega+\delta \omega) r^{*}(\omega)\right\rangle$ has been computed in that context by White et al [11] (in the limit $L \rightarrow \infty$ ) Their result was

$$
K(\delta \omega)=(2 l / c) \delta \omega \int_{0}^{\infty} d x \exp [-x(2 l / c) \delta \omega] \frac{x}{x-l}
$$

The distribution of the reflection probability $R=|r|^{2}$ through an absorbing single-mode waveguide had been studied many yeais earlier as a problem in radio-engineering [12], with the result

$$
\langle R\rangle=\left(l / c \tau_{a}\right) \int_{1}^{\infty} d z \exp \left[-(z-1)\left(l / c \tau_{a}\right)\right] \frac{z-1}{z+1}
$$

One readily veilfies that Eqs (2) and (3) are 1dentical under the substitution of $1 / \tau_{a}$ by $-\imath \delta \omega$

In a similar way one can obtain the correlator of the transmission amplitudes by analytic continuation to imaginary absorption rate of the mean transmission probability through an absorbing waveguide The absorbing problem for $N=1$ was solved by Fieılikher, Pustilnık, and Yurkevich [13] That solution will not be considered furthei here, since our interest is in the multi-mode regime, ielevant for the microwave expeiıments [5] The transmission probability in an absorbing waveguide with $N \gg 1$ is given by [14]

$$
\langle T\rangle=\frac{l}{N \xi_{\mathrm{d}} \sinh \left(L / \xi_{\mathrm{a}}\right)} \exp \left(-\delta_{\beta 1} \frac{L}{2 N l}\right),
$$

for absorption lengths $\xi_{\mathrm{a}}=\sqrt{D \tau_{a}}$ in the range $l \ll \xi_{\mathrm{a}} \ll \xi$ The length $L$ of the waveguide should be $\geqslant l$, but the relative magnitude of $L$ and $\xi$ is arbitrary Substitution of $1 / \tau_{a}$ by $-\imath \delta \omega$ gives the correlator

$$
C(\delta \omega)=\frac{l \sqrt{-l \tau_{D} \delta \omega}}{N L \sinh \sqrt{-l \tau_{D} \delta \omega}} \exp \left(-\delta_{\beta 1} \frac{L}{2 N l}\right)
$$

where $\tau_{D}=L^{2} / D$ is the diffusion time The range of validity of Eq (5) is $L / \xi \ll \sqrt{\tau_{D} \delta \omega} \ll L / l$, or equivalently $D / \xi^{2} \ll \delta \omega$ $\ll c / l$ In the diffusive regime, for $L \ll \xi$, the correlator (5) reduces to the known result [6] from pertuibation theory

Fo1 $\max \left(D / L^{2}, D / \xi^{2}\right) \ll \delta \omega \ll c / l$ the decay of the absolute value of the correlator is a stretched exponential,

$$
|C|=\frac{2 l}{N L} \sqrt{\tau_{D} \delta \omega} \exp \left(-\sqrt{\frac{1}{2} \tau_{D} \delta \omega}-\delta_{\beta 1} \frac{L}{2 N l}\right)
$$

In the localized regime, when $\xi$ becomes smaller than $L$, the onset of this tail is pushed to higher frequencies, but it retains its functional form The weight of the tail is reduced by a factor $\exp (-L / 2 N l)$ in the presence of time-reversal symmetry There is no reduction factor if time-reversal symmetry is broken

To test our analytical findings we have carried out numerical simulations The disordered medium is modeled by a two-dimensional square lattice (lattice constant $a$, length $L$, width $W$ ) The (relative) dielectric constant $\varepsilon$ fluctuates from site to site between $1 \pm \delta \varepsilon$ The multiple scattering of a scalar wave $\Psi$ (for the case $\beta=1$ ) is described by discretizing

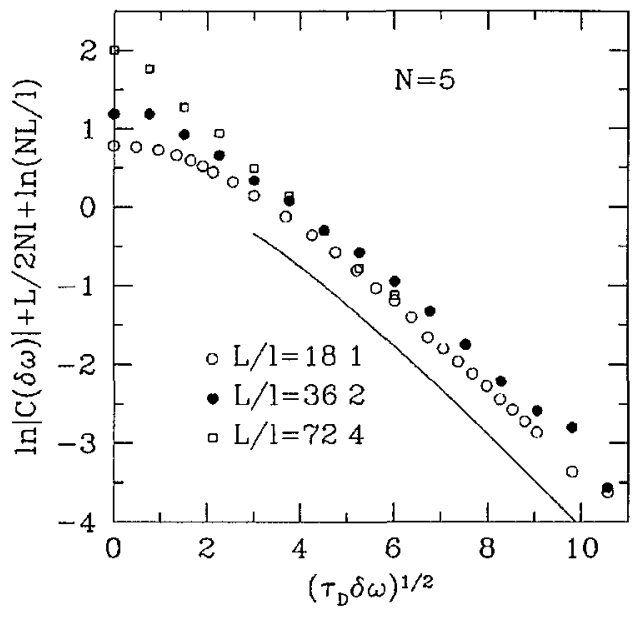

FIG 1 Frequency dependence of the logarithm of the absolute value of the correlator $C(\delta \omega)$ The data points follow from a numerical simulation for $N=5$, the solid curve is the analytical highfrequency result (6) for $N \gg 1$ (with $\beta=1$ ) The decay of the correlator is given by the diffusive time constant $\tau_{D}=L^{2} / D$ even if the length $L$ of the waveguide is greater than the localization length $\xi$ $=6 l$ The offset of about 06 between the numerical and analytical results 1 probably a finite- $N$ effect

the Helmholtz equation $\left[\nabla^{2}+(\omega / c)^{2} \varepsilon\right] \Psi=0$ and computing the tiansmission matrix using the 1ecursive Gieen function technique [15] The mean free path $l$ is determined fiom the average transmission probability $\left\langle\operatorname{Tr} t t^{\dagger}\right\rangle=N(1+L / l)^{-1}$ in the diffusive regime [8] The cortelator $C$ is obtained by averaging $t_{n m}(\omega+\delta \omega) t_{n m}^{*}(\omega)$ over the mode indices $n, m$ and over different realizations of the disorder We choose $\omega^{2}=2(c / a)^{2}, \delta \varepsilon=04$, leading to $l=221 a$ The width $W$ $=11 a$ is kept fixed (corresponding to $N=5$ ), while the length $L$ is varied in the lange $(400-1600) a$ These waveguides are well in the localized legime, $L / \xi$ 1anging from 3 to 12 A latge number (some $10^{4}-10^{5}$ ) of realizations were needed to average out the statıstical fluctuations, and this restricted our simulations to a relatively small value of $N$ For the same reason we had to limit the 1ange of $\delta \omega$ in the data set with the largest $L$

Results for the absolute value of the correlator are plotted in Fig 1 (data points) and are compared with the analytical high-fiequency prediction for $N \gg 1$ (solid cuive) We see from $F_{1} g$ that the correlators for different values of $L / \xi$ converge for large $\delta \omega$ to a curve that lies somewhat above the theoretical prediction The offset is about 06 , and could be easily explained as an $\mathcal{O}(1)$ unceitainty in the exponent in $\mathrm{Eq}$ (1) due to the fact that $N$ is not $\gg 1$ in the simulation Regardless of this offset, the simulation confiıms both analytical predictions The stretched exponential decay $\propto \exp \left(-\sqrt{\tau_{D} \delta \omega / 2}\right)$ and the exponential suppression factor $\exp (-L / 2 \xi)$ We emphasize that the time constant $\tau_{D}$ $=L^{2} / D$ of the high-frequency decay is the diffusion time for the entire length $L$ of the waveguide, even though the localization length $\xi$ is up to a factor of 12 smaller than $L$

We can summarize our findings by the statement that the correlatol of the transmission amplitudes factorizes in the high-fiequency regime $C \rightarrow f_{1}(\delta \omega) f_{2}(\xi)$ The fiequency dependence of $f_{1}$ depends on the diffusive time thiough the waveguide, even if it is longer than the localization length 
Localization has no effect on $f_{1}$, but only on $f_{2}$ We can contrast this factorization with the high-frequency asymptot1cs $K \rightarrow f_{3}(\delta \omega)$ of the correlator of the reflection amplitudes In the corresponding absorbing pioblem the high-fiequency regime conesponds to an absorption length smaller than the localization length, so it is obvious that $K$ becomes independent of $\xi$ in that regime The factorization of $C$ is less obvi- ous Since the localized regime is accessible experimentally [16], we believe that an experimental test of our piediction should be feasible

Discussions with $M$ Buttiker, L I Glazman, K A Matveev, M Pustılnık, and P G Silvestrov are gratefully acknowledged This work was supported by the Dutch Sc1ence Foundation NWO/FOM
[1] B A van Tiggelen, in Diffuse Waves in Complex Media, edited by J-P Fouque, NATO Science Series C531 (Kluwer, Dordrecht, 1999)

[2] R L Weave1, Phys Rev B 49, 5881 (1994)

[3] B White, P Sheng, and B Nair, Geophysics 55, 1158 (1990)

[4] Scattering and Localization of Classical Waves in Random Media, edited by P Sheng (World Scientıfic, Singapore, 1990)

[5] AZ Genack, P Sebbah M Stoytchev, and B A van Tiggelen, Phys Rev Lett 82, 715 (1999)

[6] R Berkovits and S Feng, Phys Rep 238, 135 (1994)

[7] The length $l=\alpha_{d} l_{\mathrm{tr}}$ differs from the transport mean free path $l_{\mathrm{tr}}$ by a dimensionality-dependent numerical coefficient $\alpha_{d}$ $=2, \pi / 2,4 / 3$ fol $d=1,2,3$ The diffusion coefficient is $D$ $=c l_{\mathrm{tr}} / d$

[8] C W J Beenakker, Rev Mod Phys 69, 731 (1997)

[9] V I Klyatskin and A I Satchev, Usp F1Z Nauk 162, 161 (1992) [Sov Phys Usp 35, 231 (1992)]

[10] This is a complication of the transmission problem The reflection problem is simpler, because the (approximate) unitaity of the reflection matrix $r$ provides additional information on the distribution of the correlator of the reflection amplitudes The mapping between the dynamic and absorbing problems has been used recently to calculate the entire distribution of the elgenvalues of $r\left(\omega+\frac{1}{2} \delta \omega\right) r^{\dagger}\left(\omega-\frac{1}{2} \delta \omega\right)$ in the limit $\delta \omega \rightarrow 0$ S A Ramakrishna and N Kumar, e-print cond-mat/9906098, CW J Beenakker and PW Brouwer, e-print cond-mat/9908325

[11] B White, P Sheng, Z Q Zhang, and G Papanicolaou, Phys Rev Lett 59, 1918 (1987)

[12] V N Tutubalın, Radıotekh Elektron 16, 1352 (1971) [Radıo Eng Electron Phys 16, 1274 (1971)], W Kohler and GC Papanicolaou, SIAM (Soc Ind Appl Math) J Appl Math 30, 263 (1976)

[13] V Freılikher, M Pustılnkk, and I Yurkevich, Phys Rev Lett 73, 810 (1994)

[14] P W Brouwer, Phys Rev B 57, 10526 (1998)

[15] H U Baranger, D P DiVincenzo, R A Jalabert, and A D Stone, Phys Rev B 44, 10637 (1991)

[16] M Stoytchev and A Z Genack, Opt Lett 24, 262 (1999) 